\title{
Revisión Sistemática sobre salud digital en la gestión del absentismo y el retorno al trabajo
}

Systematic review of the role of digital health in managing sickness absence and return to work

Gema Arevalo Alonso ${ }^{1}$ (]) 0000-0002-8909-3308

${ }^{1}$ Federation of Occupational Health Nurses within the EU (FOHNEU), Milton Keynes, Reino Unido.

Fechas · Dates

Recibido: 05/12/2021

Aceptado: 12/01/2022

Publicado: 17/01/2022
Correspondencia · Corresponding Author

Gema Arevalo Alonso

Federation of Occupational Health Nurses within the EU (FOHNEU), Reino Unido E-mail: gemaarevalo@enfermeriadeltrabajo.com 


\section{Resumen}

Objetivo: El uso de tecnologías digitales se ha convertido en una estrategia para incrementar la efectividad de los servicios de salud. El objetivo de este estudio es analizar el impacto del uso de las herramientas de salud digital sobre la gestión del absentismo laboral y el retorno al trabajo.

Métodos: Se realizó una revisión sistemática siguiendo el formato PRISMA en las bases de datos electrónicas Pubmed, Cochrane, Web of Science, CINAHL y ScienceDirect. La calidad metodológica fue analizada a partir de las herramientas de evaluación crítica del Joanna Briggs Institute (JBI).

Resultados: Un total de 24 estudios fueron incluidos en esta revisión, de los cuales se extrajeron resultados muy heterogéneos debido a la variabilidad de métodos de medición. El uso de herramientas digitales contribuía, mayoritariamente, en la mejora de las cifras de absentismo y de retorno a la actividad laboral, no evidenciándose en ninguno que ocasionara un perjuicio. Adicionalmente, en 10 de los estudios se obtuvieron datos sobre presentismo y otros factores influyentes en el absentismo y el retorno a la actividad laboral, como fueron la resiliencia, el compromiso laboral y el desapego psicológico al trabajo.

Conclusiones: El uso de herramientas de salud digital contribuye positivamente en la recuperación de los trabajadores que se encuentran en situación de incapacidad temporal, facilitando la gestión del retorno al trabajo y, por consiguiente, favoreciendo la reducción del absentismo laboral.

Palabras clave: Telemedicina; Tecnología digital; Reinserción al trabajo; Absentismo; Salud laboral; Enfermería del trabajo; Enfermedad crónica.

\section{Abstract}

Objective: Digital technologies have become a strategy to improve the effectiveness of health services. The objective of this study was to analyse the impact of the use of digital health tools on the management of sickness absence and return to work.

Methods: A systematic review was conducted in accordance with the PRISMA guidelines. The following databases were searched for the period April 2021 to June 2021: Pubmed, Cochrane, Web of Science, CINAHL and ScienceDirect. Methodological quality was assessed by using the Joanna Briggs Institute (JBI) critical appraisal tools.

Results: A total of 24 studies were eligible for inclusion; there was great heterogeneity of results, due to a wide range of measurement methods. The majority of outcomes supported the use of digital tools as making an important contribution to in managing the number of sickness absence and return to work cases. In some studies, there were no significant changes as compared to traditional case management; nevertheless, none of the 24 studies found detrimental effects due to the use of digital health tools. Additionally, 10 of the studies provided data on presenteeism and other determinants of $\backslash$ sickness absence and return to work, including resilience, work engagement and psychological detachment from work. 
Conclusions: The use of digital health tools contributes positively to the recovery of workers who are in a situation of temporary disability, facilitating the management of return to work and favoring a decrease in workplace sickness absence.

Key words: telemedicine; digital technology; return to work; absenteeism; sickness absence; occupational health; occupational health nursing; chronic disease.

\section{Introducción}

La pandemia Covid-19 ha sido un acelerador para la implantación de herramientas digitales en la práctica sanitaria, que han resultado de gran utilidad para facilitar el trabajo de los profesionales y para garantizar la continuidad de la atención ${ }^{(3)}$.

Los especialistas en salud laboral se enfrentan al reto y necesidad de ir en línea con las tendencias económicas, los cambios en los patrones de empleo y, por consiguiente, con los riesgos y enfermedades que emergen de ellos. Su finalidad es maximizar la salud en el entorno laboral, a través de la implementación de estrategias que mejoren la salud, la participación y responsabilidad de los trabajadores, haciendo uso de la tecnología y promocionando ambientes de trabajo saludables ${ }^{(4)}$, dando solución a los cada vez más complejos problemas de salud y seguridad en el entorno laboral y haciendo uso de competencias esenciales, como la gestora ${ }^{(5)}$. El trabajo se considera como un factor importante en la participación laboral de la sociedad, siendo las enfermedades crónicas uno de los principales factores de salud que tienen un efecto sobre dicha participación, por contribuir a la reducción de la productividad laboral y la probabilidad de retiro temprano con el consiguiente impacto sobre el mercado laboral, la economía y los costes sanitarios ${ }^{(6)}$.

La vuelta o retorno al trabajo es un momento que debe gestionarse y planificarse con suficiente antelación y debe basarse en las necesidades específicas del trabajador y la capacidad de la empresa para responder a las mismas. Los programas de gestión de retorno al trabajo que se llevan a cabo en las empresas contribuyen positivamente en la calidad de vida, la reincorporación sociolaboral de los individuos y, por ende, en las cifras de absentismo(7,8).

El absentismo tiene un impacto negativo para la empresa con independencia de la causa. Según los datos del VIII Informe de absentismo Adecco, en 2018, la pérdida total de horas de trabajo llegó a casi 1.350 millones que, en número de personas, correspondería a 753.000 asalariados que no trabajaron en todo el año(9). Por otra parte, el presentismo laboral, además de afectar la productividad directa de cada trabajador, lo hace de forma indirecta contra la organización para la que trabaja(10).

La digitalización de la salud en el lugar de trabajo ofrece un gran potencial, pero no cabe duda que debemos asegurar que su aplicación sea adecuada, responsable y segura y no suponga ningún perjuicio para el usuario, ya sea paciente o profesional. Debido a las múltiples herramientas disponibles en el mercado y con el propósito de evitar la elección inadecuada por parte de profesionales y usuarios, 
algunas organizaciones han publicado guías o listados para estandarizar aquellas de las que se tiene evidencia sobre su efectividad(11-13).

La literatura nos muestra evidencia de los resultados positivos que proporcionan las herramientas digitales aplicadas en salud, sin embargo, el rápido avance de las tecnologías lleva a la necesidad de tener que realizar nuevos estudios y actualizar los datos sobre nuevas herramientas y sus posibles efectos ${ }^{(3,14)}$.

Existen revisiones sistemáticas que tratan la salud digital en el entorno laboral enfocadas a la promoción y prevención de la salud, en cambio, serían necesarias revisiones enfocadas a otras áreas de la salud laboral, como la puramente gestora ${ }^{(15-18)}$.

El objetivo de esta revisión sistemática es analizar el efecto de las herramientas digitales en la gestión de absentismo laboral y en el retorno al trabajo, así como conocer los resultados sobre el presentismo $\mathrm{u}$ otros factores relacionados con la salud laboral. Se espera que la implantación de un sistema de salud digital en la empresa supondría una mejora para todas las partes implicadas, contribuyendo al fortalecimiento del sistema. Sin embargo, debemos tener evidencia de su nivel de efectividad en todas las áreas que la salud laboral engloba, en especial, el área de gestión.

\section{Métodos}

\section{Diseño del estudio}

Se realizó una búsqueda sistemática de estudios, siguiendo los criterios de la Declaración PRISMA ${ }^{(1)}$ que proporcionaran resultados sobre el uso de herramientas digitales en intervenciones sanitarias y su impacto sobre la gestión del absentismo y el retorno al trabajo tras una situación de incapacidad temporal. Haciendo uso del formato PICO se formuló la pregunta de investigación de la cual se obtuvieron las palabras claves utilizadas en la búsqueda como se muestra en la Tabla 1.

Tabla 1: Formato PICO: palabras clave.

\begin{tabular}{ll}
\hline Población & Población trabajadora \\
\hline Intervención & Uso de las herramientas de salud digital \\
\hline Comparación & Métodos tradicionales \\
\hline Outcomes / Resultados & Impacto en los resultados de absentismo por incapacidad \\
& temporal y en el retorno a la actividad laboral \\
\hline Pregunta de investigación & $\begin{array}{l}\text { El uso de herramientas de salud digital en la población } \\
\text { trabajadora ¿mejora los resultados de gestión del absentismo } \\
\text { por incapacidad temporal y de retorno a la actividad laboral? }\end{array}$ \\
\hline
\end{tabular}

Fuente: elaboración propia. 


\section{Estrategia de la búsqueda}

La búsqueda se realizó en las bases de datos electrónicas PubMed, Web of Science, Cochrane, Google Scholar and CINALH a partir de las palabras clave resultantes de la pregunta en formato PICO, tal como se expresa en la Tabla 2.

Tabla 2: Términos empleados para la búsqueda.

\begin{tabular}{|c|c|c|c|}
\hline MeSH & $\begin{array}{l}\text { Operador } \\
\text { booleano }\end{array}$ & Términos libres & $\begin{array}{l}\text { Operador } \\
\text { booleano }\end{array}$ \\
\hline Telemedicine & OR & $\begin{array}{l}\text { telemedicine or mobile health or mhealth or } \\
\text { telehealth or ehealth }\end{array}$ & OR \\
\hline Wireless Technology & & & OR \\
\hline Smartphone & OR & smartphone or smartphones & OR \\
\hline $\begin{array}{l}\text { Decision Making, } \\
\text { Computer-Assisted }\end{array}$ & & & AND \\
\hline Occupational Health & OR & occupational health & OR \\
\hline Workplace & OR & workplace or workplaces & OR \\
\hline Workforce & OR & $\begin{array}{l}\text { workforce or workforces or employee or } \\
\text { employees }\end{array}$ & OR \\
\hline Return to Work & OR & return to work or back to work & OR \\
\hline Absenteeism & OR & absenteeism & OR \\
\hline Sick Leave & OR & sick leave or sick days or sick day & \\
\hline
\end{tabular}

Se utilizó la web de la National Library of Medicine para identificar los descriptores de búsqueda del Medical Subject Headings (MeSH) ${ }^{(19)}$. Los descriptores seleccionados fueron: telemedicine, wireless technology, smartphone, decision making computer-assisted, occupational health, workplace, workforce, return to work, absenteeism y sick leave. Con el objetivo de mejorar el alcance de los resultados y de completar la búsqueda en base a los descriptores MeSH, se usaron términos sinónimos. Se emplearon los operadores booleanos AND y OR para la unión de términos. 
El proceso de búsqueda se llevó a cabo entre los meses de abril y junio de 2021. La Tabla 3 muestra la estrategia de búsqueda para cada una de las bases de datos utilizadas, de las cuales se obtuvieron 20 estudios para su análisis posterior.

Tabla 3: Estrategia de búsqueda.

\begin{tabular}{|c|c|c|c|c|}
\hline $\begin{array}{l}\text { Base de } \\
\text { datos }\end{array}$ & Ecuación de búsqueda & $\begin{array}{l}\text { Fecha de bús- } \\
\text { queda }\end{array}$ & $\begin{array}{l}\text { Resulta- } \\
\text { dos }\end{array}$ & $\begin{array}{l}\text { Selecciona- } \\
\text { dos }\end{array}$ \\
\hline Pubmed & $\begin{array}{l}\text { (Telemedicine[Mesh] OR Telemedicine[Title/ } \\
\text { Abstract] OR Mobile Health[Title/Abstract] OR } \\
\text { mHealth[Title/Abstract] OR Telehealth[Title/Abs- } \\
\text { tract] OR eHealth[Title/Abstract] OR Wireless } \\
\text { Technology[Mesh] OR Smartphone[Mesh] } \\
\text { OR Smartphone[Title/Abstract] OR Smar- } \\
\text { tphones[Title/Abstract] OR Decision Making, } \\
\text { Computer-Assisted[Mesh] ) AND (Occupational } \\
\text { Health[Mesh] OR Occupational Health[Title/Abs- } \\
\text { tract] OR Workplace[Mesh] OR Workplace[Title/ } \\
\text { Abstract] OR Workplaces[Title/Abstract] OR } \\
\text { Workforce[Mesh] OR Workforce[Title/Abstract] } \\
\text { OR Workforces[Title/Abstract] OR employee[- } \\
\text { Title/Abstract] OR employees[Title/Abstract] OR } \\
\text { Return to Work[Mesh] OR Return to Work[Title/ } \\
\text { Abstract] OR Back to Work[Title/Abstract] OR } \\
\text { absenteeism[Mesh] OR absenteeism[Title/Abs- } \\
\text { tract] OR Sick Leave[Mesh] OR Sick Leave[Title/ } \\
\text { Abstract] OR Sick Days[Title/Abstract] OR Sick } \\
\text { Day[Title/Abstract] ) AND ('2015/O1/O1'[PDat] : } \\
\text { '2021/04/24'[PDat]) }\end{array}$ & $25 / 04 / 2021$ & 1.007 & 17 \\
\hline $\begin{array}{l}\text { Web of } \\
\text { science }\end{array}$ & $\begin{array}{l}\text { TEMA: ( Telemedicine OR Videoconferencing } \\
\text { OR Wireless Technology OR Smartphone OR } \\
\text { Decision Making Computer Assisted) AND } \\
\text { TEMA: (Occupational Health OR Workplace OR } \\
\text { Workforce OR employee OR employees) AND } \\
\text { TEMA: ( Return Work OR absenteeism OR Sick } \\
\text { Leave) } \\
\text { Filtrado por: Año 2015-2021 }\end{array}$ & 25/04/2021 & 46 & 6 \\
\hline Cochrane & $\begin{array}{l}\text { ( Telemedicine OR ehealth OR mhealth) AND ( } \\
\text { Occupational Health OR Workplace OR emplo- } \\
\text { yee ) AND (Return Work OR absenteeism ) } \\
\text { Filtrado por research articles, Medicine and } \\
\text { Dentistry, Nursing and Health Professions } \\
\text { Filtrado por: Año 2015-2021 }\end{array}$ & 25/04/2021 & 23 & 7 \\
\hline $\begin{array}{l}\text { Google } \\
\text { Scholar }\end{array}$ & $\begin{array}{l}\text { "occupational health" OR workplace OR workfor- } \\
\text { ce OR employee AND telemedicine OR "mobile } \\
\text { health" OR mhealth OR telehealth OR ehealth } \\
\text { AND "return to work" OR "back to work" OR } \\
\text { absenteeism } \\
\text { Filtrado por: Año } 2021\end{array}$ & $25 / 04 / 2021$ & 705 & 4 \\
\hline CINALH & $\begin{array}{l}\text { ehealth (AB Resumen) AND return to work (AB } \\
\text { Resumen) OR absenteeism (AB Resumen) AND } \\
\text { workplace (AB Resumen) } \\
\text { Filtrado por: } 2016 \text { - } 2021 \\
\text { Filtrado por: publicaciones académicas }\end{array}$ & 28/06/2021 & 160 & 2 \\
\hline \multicolumn{3}{|c|}{ TOTAL / TOTAL (después de eliminar duplicados) } & 1.918 & $36 / 20$ \\
\hline
\end{tabular}




\section{Criterios de selección}

Para la selección de los estudios se utilizaron los siguientes criterios:

\section{Criterios de inclusión:}

- Artículos publicados en idioma español y/o inglés.

- Artículos publicados entre el periodo 2015-2021.

- Tipología: artículos originales, reporte de casos y comunicación breve/corta. Se valoraron especialmente los ensayos controlados aleatorizados por su fiabilidad como evidencia científica.

- Estudios realizados por profesionales del área sanitaria (enfermería, medicina, fisioterapia, terapia ocupacional.)

- Artículos que analizaran alguno de los siguientes valores: índices/cifras de absentismo, días de ausencia al trabajo y relacionados con el retorno al trabajo o la reincorporación a la actividad laboral.

- Estudios referidos a la población trabajadora activa (16-65 años) de todos los sexos.

\section{Criterios de exclusión:}

- Artículos de baja calidad científico-técnica tras aplicar las herramientas de evaluación de la calidad específicas para cada tipo de articulo.

- Artículos que no dieran respuesta a la pregunta de investigación y a los objetivos de la presente revisión sistemática.

- Tipología: artículos de opinión, editoriales, cartas al director/editor

\section{Evaluación de la calidad metodológica}

Se determinó la calidad metodológica de los estudios seleccionados mediante las herramientas de evaluación critica proporcionadas por el Joanna Briggs Institute (JBI) para cada tipo de diseño(2).

Se utilizaron las diferentes versiones de listas de comprobación o checklist para ensayos controlados aleatorios (13 ítems), estudios de cohortes (12 ítems), estudios cualitativos (10 ítems), estudios cuantitativos transversales (8 ítems) y evaluaciones económicas (11 ítems). El punto de corte que se estableció para cada tipo de estudio fue de un $70 \%$ sobre la puntuación total obtenida de cada checklist. 


\section{Resultados y discusión}

Las estrategias de búsqueda identificaron un total de 1.955 referencias, las cuales fueron sometidas a sucesivos cribados, tal como se presenta en la Figura 1.

\section{Figura 4}

Resultados de la búsqueda (Diagrama de flujo - PRISMA)

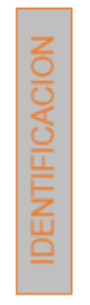

Referencias identificadas: 1.946

Pubmed: 1.007, WOS: 46, Cochrane: 23 ,

Google Scholar: 705, CINALH: 160

Referencias identificadas a

través de otros recursos: 9

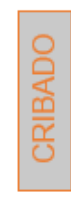

Referencias cribadas tras eliminar duplicados

$(n=1.910)$
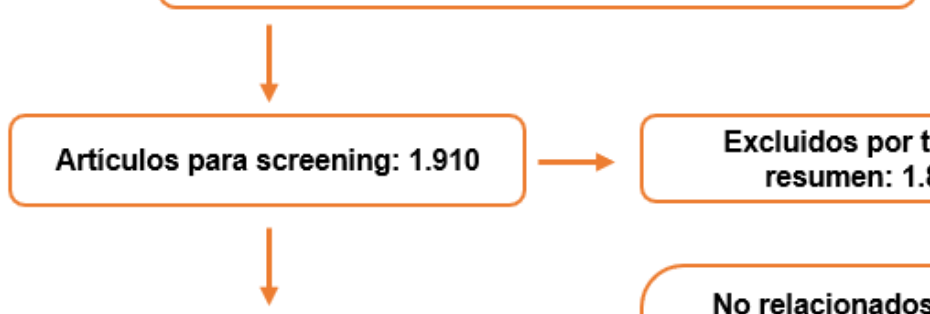

Artículos a texto completo: 70

No relacionados con el objetivo: 25
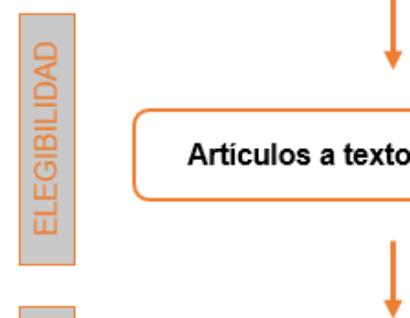

No finalizado: 1

No datos relevantes: 8

Tipologia: 11

Versión anterior: 1

\section{Artículos incluidos en la revisión:}

24

Figura 1: Diagrama de flujo de los resultados de la búsqueda.

Finalmente se seleccionaron 24 estudios, de los cuales 15 fueron ensayos controlados aleatorios, 3 estudios de cohortes, 3 estudios cualitativos, 2 estudios cuantitativos y 1 evaluación económica. Los registros obtenidos fueron exportados al gestor de referencias bibliográficas Mendeley ${ }^{(20)}$.

Se valoraron los veinticuatro estudios a través de las herramientas proporcionadas por el Joanna Briggs Institute $(\mathrm{JBI})^{(2)}$ siendo las puntuaciones obtenidas en todos ellos, intermedias-altas, tal como se presenta en la Tabla 4. 
Tabla 4: Resultados de la evaluación critica de artículos.

\begin{tabular}{|c|c|c|}
\hline Tipo estudio & Estudios evaluados (Autor, año) & Puntuación \\
\hline \multirow{3}{*}{$\begin{array}{l}\text { Ensayo } \\
\text { controlado } \\
\text { aleatorio }\end{array}$} & $\begin{array}{l}\text { Suman et al, (2019)(21); Van der Meij et al, (2018) }{ }^{(22) ;} \text {; Blanquero } \\
\text { et al, (2020)(23) }\end{array}$ & $12 / 13$ \\
\hline & $\begin{array}{l}\text { Deady et al, (2020)(24); Lokman et al, }(2017)^{(25)} \text {; Van der Hout et } \\
\text { al, (2020)(26) }\end{array}$ & $11 / 13$ \\
\hline & $\begin{array}{l}\text { Notenbomer et al, (2018)(27); Volker et al, (2015)(28); Hutting et } \\
\text { al, (2015)(29); Ebert et al, (2016)(30); Bouwsma et al, (2017) }{ }^{(31) ;} \\
\text { Beiwinkel et al, (2017) }{ }^{(32) ;} \text {; Ghoshchi et al, (2020) }{ }^{(33)} \text {; Wolf et al, } \\
(2016)^{(34) ;} \text { de Jong et al, (2020)(35) }\end{array}$ & $10 / 13$ \\
\hline $\begin{array}{l}\text { Estudio de } \\
\text { cohortes }\end{array}$ & $\begin{array}{l}\text { Schaetz et al, (2020)(36); Mochari-Greenberger et al, (2020)(37); } \\
\text { Anderson et al, (2019)(38) }\end{array}$ & $10 / 12$ \\
\hline \multirow[t]{3}{*}{ Estudio cualitativo } & Engdahl et al, (2020)(39) & $10 / 10$ \\
\hline & Beaton et al, $(2020)^{(40)}$ & $9 / 10$ \\
\hline & Gross et al, $(2020)^{(41)}$ & $8 / 10$ \\
\hline $\begin{array}{l}\text { Estudio } \\
\text { cuantitativo }\end{array}$ & Collins et al $(2020)^{(42)}$ & $7 / 10$ \\
\hline $\begin{array}{l}\text { Estudio } \\
\text { cuantitativo } \\
\text { transversal }\end{array}$ & Karpman et al, (2019)(43) & $7 / 8$ \\
\hline $\begin{array}{l}\text { Evaluación } \\
\text { económica }\end{array}$ & Noben et al, (2015)(44) & $9 / 11$ \\
\hline
\end{tabular}

La procedencia de los artículos seleccionados fue de 9 países. Se encontraron 10 estudios realizados en los Países Bajos, 3 en Canadá, 2 en Australia y 2 en EEUU, correspondiendo el resto de estudios a países europeos como España, Italia, Alemania, Suiza y Suecia.

Del total de artículos seleccionados, en 16 de ellos la muestra estuvo formada únicamente por trabajadores, tres estudios, además, integraron pacientes, en dos se incluyeron profesionales y en uno de ellos se incorporó a los familiares (laboralmente activos) de los trabajadores.

La Tabla 6 muestra las características de cada uno de los estudios seleccionados. Fueron clasificados por autor y año de publicación, tipo de estudio, contexto y país, objetivo, participantes, métodos y principales hallazgos. 
Tabla 5: Características de los estudios incluidos en la revisión sistemática

\begin{tabular}{llll}
\hline $\begin{array}{l}\text { Autor, tipo } \\
\text { estudio, } \\
\text { contexto }\end{array}$ & Objetivo del estudio & Participantes & Métodos \\
\hline Deady et al, & Evaluar la efectividad & - Grupo intervención & - HPQ: productividad \\
$2020(24)$ & de una aplicación para & $(\mathrm{n}=1128)$ & laboral a través \\
Ensayo & teléfonos inteligentes & EM 40.20 (DS 10.88) & del cálculo de días \\
controlado & diseñada para reducir los & $72,4 \%$ Hombres & laborales efectivos \\
aleatorio. & síntomas de depresión & - Grupo atención & uniendo los datos \\
Sydney & yla aparición de nuevos & habitual (n=1143) & de absentismoy \\
(Australia) & casos en un grupo de & EM 40.32 (DS 10.38) & presentismo. \\
& trabajadores australianos. & $76.0 \%$ Hombres & - CD-RISC10: \\
& & & resiliencia.
\end{tabular}

Principales hallazgos contexto

En el grupo esalud se observó una diferencia significativa en el rendimiento laboral (HPQ) durante el seguimiento. $\left[t_{(935,4)}=3,03, p=0,003\right]$ (equivaldría a 1 día adicional de trabajo efectivo cada mes, lo cual es un beneficio funcional y económico considerable). Además se observó una mejora del nivel de resiliencia durante el seguimiento $\left[\mathrm{t}_{(464.10)}=3.01\right.$, $p=0.003$ ]

\begin{tabular}{llll}
\hline Lokman et al, & Evaluar los beneficios & - Grupo intervención & - TiC-P \\
$2017(25)$ & y costes para la salud & $(n=131)$ & - ROl \\
Ensayo & y la economía de una & EM 43.3 (DS 9.5) & - Análisis de costes \\
controlado & intervención guiada de & 58.8\% Mujeres & de pérdida de \\
aleatorio & eSalud con el objetivo & - Grupo atención & productividad debido \\
de dos brazos. & de que los empleados & habitual (n=89) & al absentismoy \\
Pequeñas & que figuran en la lista de & EM 45.5 (DS 10.7) & presentismo \\
y medianas & absentismo tengan un RT & $59.6 \%$ Mujeres & (en euros 2011). \\
empresas & más rápido. & &
\end{tabular}

En el grupo de esalud se evidenció una reducción del absentismo. El presentismo aumento en los primeros 9 meses debido a un RT temprano. Sin embargo, a partir del mes 9 disminuyó debido a la mejora en la calidad de vida que experimentan las personas al desempeñar un trabajo. Como resultado global se redujo el coste asociado al absentismo y presentismo en el grupo de eSalud (3486 €) y un ROI positivo de 10.6 euros/euro invertido.

\begin{tabular}{|c|c|c|c|c|}
\hline $\begin{array}{l}\text { Notenbomer et } \\
\text { al, } 2018^{(27)} \\
\text { Ensayo } \\
\text { controlado } \\
\text { aleatorio de } \\
\text { tres brazos. } \\
\text { (Países bajos) }\end{array}$ & $\begin{array}{l}\text { Evaluar el efecto de } \\
\text { una intervención de } \\
\text { esalud para reducir la } \\
\text { frecuencia de absentismo } \\
\text { por enfermedad de } \\
\text { los trabajadores con } \\
\text { absentismo frecuente, } \\
\text { comparado con el } \\
\text { cuidado tradicional. }\end{array}$ & $\begin{array}{l}\text { - Grupo eSalud } \\
(\mathrm{n}=21) \text {, } \\
\text { EM 44.9 (DS 10.1) } \\
\text { 33\% Hombres } \\
\text { - Grupo eSalud + } \\
\text { consulta médico del } \\
\text { trabajo (n=31) } \\
\text { EM } 45.9 \text { (DS 11.4) } \\
\text { 32\% Hombres } \\
\text { - Grupo atención } \\
\text { habitual (n=30) } \\
\text { EM } 46.9 \text { (DS 10.9) } \\
\text { 33\% Hombres }\end{array}$ & $\begin{array}{l}\text { - Cuestionario de } \\
\text { escala validada } \\
\text { con preguntas } \\
\text { sobre factores } \\
\text { determinantes } \\
\text { de absentismo y } \\
\text { preguntas abiertas } \\
\text { sobre frecuencia de } \\
\text { absentismo. } \\
\text { - UWES } \\
\text { - WAl }\end{array}$ & $\begin{array}{l}\text { Ambos grupos de } \\
\text { intervención eSalud y } \\
\text { eSalud + consulta con } \\
\text { el médico, mostraron } \\
\text { una reducción en días } \\
\text { de absentismo pero sin } \\
\text { diferencias significativas } \\
\text { entre ambos. } \\
\text { No se encontró ningún } \\
\text { resultado significativo } \\
\text { de la intervención de } \\
\text { eSalud como herramienta } \\
\text { independiente para } \\
\text { reducir la frecuencia de } \\
\text { ausencias por enfermedad } \\
\text { o aumentar el compromiso } \\
\text { y la capacidad laboral. }\end{array}$ \\
\hline
\end{tabular}




\begin{tabular}{|c|c|c|c|c|}
\hline $\begin{array}{l}\text { Autor, tipo } \\
\text { estudio, } \\
\text { contexto }\end{array}$ & Objetivo del estudio & Participantes & Métodos & Principales hallazgos \\
\hline $\begin{array}{l}\text { Volker et al, } \\
2015(28) \\
\text { Ensayo } \\
\text { controlado } \\
\text { aleatorio de } \\
\text { dos grupos. } \\
\text { Pequeñas } \\
\text { y medianas } \\
\text { empresas } \\
\text { (Países bajos) }\end{array}$ & $\begin{array}{l}\text { Evaluar el efecto de una } \\
\text { intervención combinada } \\
\text { de esalud frente a la } \\
\text { atención habitual para el } \\
\text { RT de los empleados en IT } \\
\text { por trastornos mentales } \\
\text { comunes. }\end{array}$ & $\begin{array}{l}\text { - Grupo intervención } \\
(n=131) \\
\text { EM 43.4 (DS 9.5) } \\
\text { 58.8\% Mujeres } \\
\text { - Grupo atención } \\
\text { habitual ( } n=89) \\
\text { EM 45.5 (DS 10.7) } \\
60 \% \text { Mujeres }\end{array}$ & $\begin{array}{l}\text { - Cálculo de } \\
\text { días de ausencia } \\
\text { hasta el primer RT o } \\
\text { RT completo. } \\
\text { - TiC-P }\end{array}$ & $\begin{array}{l}\text { En el primer RT (parcial } \\
\text { o total), de promedio, los } \\
\text { participantes del grupo } \\
\text { intervención regresaron } \\
27 \text { días antes que los del } \\
\text { grupo de control. } \\
\text { Sin embargo, en el RT } \\
\text { completo no se apreció } \\
\text { diferencia entre los grupos. }\end{array}$ \\
\hline $\begin{array}{l}\text { Hutting et al, } \\
2015^{(29)} \\
\text { Ensayo } \\
\text { controlado } \\
\text { aleatorio. } \\
\text { (Nijmegen, } \\
\text { Países bajos) }\end{array}$ & $\begin{array}{l}\text { Evaluar la efectividad } \\
\text { de una intervención } \\
\text { autogestionada que } \\
\text { incluye eSalud, en } \\
\text { comparación con la } \\
\text { atención habitual, en } \\
\text { empleados con molestias } \\
\text { crónicas inespecíficas del } \\
\text { brazo, cuello u hombro. }\end{array}$ & $\begin{array}{l}\text { - Grupo intervención } \\
(n=66) \\
\text { EM 44.98 (DS 11.15) } \\
\text { 82.8\% Mujeres } \\
\text { - Grupo atención } \\
\text { habitual ( } n=57) \\
\text { EM 47.69 (DS 10.50) } \\
\text { 67.9\% Mujeres }\end{array}$ & $\begin{array}{l}\text { - Cálculo de días de } \\
\text { ausencia en el último } \\
\text { mes. } \\
\text { - SPS-6 }\end{array}$ & $\begin{array}{l}\text { Los datos de absentismo } \\
\text { mejoraron, sin embargo, no } \\
\text { hubo cambios significativos } \\
\text { en relación a la capacidad } \\
\text { para el trabajo. } \\
\text { Presentismo: la } \\
\text { intervención con eSalud } \\
\text { mejoró la capacidad para } \\
\text { asumir la discapacidad en } \\
\text { el trabajo. } \\
\text { Según el autor los } \\
\text { resultados deben } \\
\text { interpretarse con } \\
\text { precaución. }\end{array}$ \\
\hline $\begin{array}{l}\text { Ebert et al, } \\
2016^{(30)} \\
\text { Ensayo } \\
\text { controlado } \\
\text { aleatorio de } \\
\text { dos brazos. } \\
\text { Compañía } \\
\text { seguros } \\
\text { (Alemania) }\end{array}$ & $\begin{array}{l}\text { Investigar la aceptabilidad, } \\
\text { eficacia y resultados } \\
\text { relacionados con } \\
\text { el trabajo de una } \\
\text { intervención digital (iSMI) } \\
\text { en trabajadores con } \\
\text { niveles elevados de estrés. }\end{array}$ & $\begin{array}{l}\text {-Grupo intervención } \\
(n=131) \\
\text { EM 41(DS 9) } \\
74 \% \text { Mujeres } \\
\text {-Grupo atención } \\
\text { habitual (n=132) } \\
\text { EM } 42 \text { (DS 9) } \\
69 \% \text { Mujeres }\end{array}$ & $\begin{array}{l}\text { - UWES } \\
\text { - REQ-PD } \\
\text { - TIC-P }\end{array}$ & $\begin{array}{l}\text { Se encontraron mejoras } \\
\text { relativas al desapego } \\
\text { psicológico respecto al } \\
\text { trabajo y el presentismo, } \\
\text { sin embargo, no hubo } \\
\text { diferencias significativas en } \\
\text { el compromiso laboral y el } \\
\text { absentismo. }\end{array}$ \\
\hline $\begin{array}{l}\text { Van der Hout et } \\
\text { al, } 2020(26) \\
\text { Ensayo } \\
\text { controlado } \\
\text { aleatorio. } \\
14 \text { hospitales } \\
\text { (Países bajos) }\end{array}$ & $\begin{array}{l}\text { Evaluar el coste utilidad } \\
\text { del programa de eSalud } \\
\text { Oncokompas comparado } \\
\text { con la atención habitual } \\
\text { en supervivientes de } \\
\text { cáncer. }\end{array}$ & $\begin{array}{l}\text { - Grupo intervención } \\
(n=320) \\
\text { (38\% trabajadores) } \\
\text { EM } 65 \text { (entre } 56-71) \\
51 \% \text { Hombres } \\
\text { - Grupo atención } \\
\text { habitual ( } n=305) \\
\text { (33\% trabajadores) } \\
65 \text { (entre 57-71) } \\
48 \% \text { Hombres }\end{array}$ & $\begin{array}{l}\text { - iPCQ } \\
\text {-Cálculo de costes } \\
\text { (incluidos absentismo } \\
\text { y presentismo). }\end{array}$ & $\begin{array}{l}\text { Los costes totales medios } \\
\text { en el grupo de intervención } \\
\text { fueron más bajos, aunque } \\
\text { no significativos, que los } \\
\text { costes totales medios en el } \\
\text { grupo de control (los costes } \\
\text { incrementales fueron: - } \\
163 € \\
\text { (IC del } 95 \% \text {, - } 665 \text { € a } 326 \text { €) }\end{array}$ \\
\hline
\end{tabular}




\begin{tabular}{|c|c|c|c|c|}
\hline $\begin{array}{l}\text { Autor, tipo } \\
\text { estudio, } \\
\text { contexto }\end{array}$ & Objetivo del estudio & Participantes & Métodos & Principales hallazgos \\
\hline
\end{tabular}

\begin{tabular}{|c|c|c|c|c|}
\hline $\begin{array}{l}\text { Bouwsma } \\
\text { et al, } 2017(31) \\
\text { Ensayo } \\
\text { controlado } \\
\text { aleatorio } \\
\text { de grupos } \\
\text { escalonados. } \\
9 \text { hospitales } \\
\text { (Países Bajos) }\end{array}$ & $\begin{array}{l}\text { Evaluar la implementación } \\
\text { y efectividad de un } \\
\text { programa de atención } \\
\text { preoperatoria basado en } \\
\text { Internet para mujeres } \\
\text { trabajadoras sometidas } \\
\text { a cirugía ginecológica por } \\
\text { enfermedad benigna. }\end{array}$ & 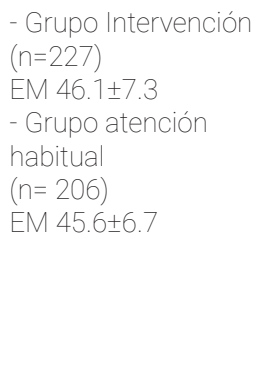 & $\begin{array}{l}\text { - Duración de } \\
\text { incapacidad por } \\
\text { IT hasta el RT } \\
\text { completo }{ }^{\star \star} \text {. }\end{array}$ & $\begin{array}{l}\text { El programa demostró un } \\
\text { gran potencial para acelerar } \\
\text { la recuperación y mejorar } \\
\text { las tasas de RT tras la } \\
\text { realización de diferentes } \\
\text { cirugías ginecológicas. } \\
\text { En el grupo de intervención } \\
\text { la media de tiempo hasta el } \\
\text { RT completo fue de } 49 \text { días } \\
\text { mientras que en el grupo de } \\
\text { control fue de } 62 \text { días. }\end{array}$ \\
\hline
\end{tabular}

\begin{tabular}{|c|c|c|c|c|}
\hline $\begin{array}{l}\text { Suman et al, } \\
2019(21) \\
\text { Ensayo } \\
\text { controlado } \\
\text { aleatorio por } \\
\text { grupos. } \\
\text { Atención } \\
\text { primaria. } \\
\text { (Ámsterdam, } \\
\text { Países bajos) }\end{array}$ & $\begin{array}{l}\text { Evaluar la eficacia y } \\
\text { la rentabilidad de una } \\
\text { estrategia de eSalud } \\
\text { multifacética para mejorar } \\
\text { la percepción del dolor y } \\
\text { reducir la discapacidad } \\
\text { y el absentismo en } \\
\text { pacientes con dolor de } \\
\text { espalda. }\end{array}$ & $\begin{array}{l}\text { - Grupo intervención } \\
(n=331) \\
\text { (Trabajadores n= } \\
\text { 183) } \\
\text { EM 55.7 (DS 13.9) } \\
59 \% \text { Mujeres } \\
\text { - Grupo atención } \\
\text { habitual ( } n=448 \text { ) } \\
\text { (Trabajadores } \\
\text { n=232) EM 56.6 (DS } \\
\text { 14.6) } \\
57 \% \text { Mujeres }\end{array}$ & $\begin{array}{l}\text { - TIC-P } \\
\text { - PRODISQ }\end{array}$ & $\begin{array}{l}\text { La estrategia de eSalud } \\
\text { multifacética no fue eficaz } \\
\text { para mejorar la percepción } \\
\text { de los pacientes sobre el } \\
\text { dolor de espalda o para } \\
\text { disminuir la discapacidad y } \\
\text { el absentismo. } \\
\text { La media de días de } \\
\text { absentismo } \\
\text { en grupo Intervención } \\
\text { fue } 0.7(2.7) \text { y en el grupo } \\
\text { control fue de } 0.7(4.4) \text {. }\end{array}$ \\
\hline $\begin{array}{l}\text { Van der Meij } \\
\text { et al, } \\
2018^{(22)} \\
\text { Ensayo } \\
\text { controlado } \\
\text { aleatorio, } \\
\text { multicéntrico, } \\
\text { simple ciego. } \\
7 \text { hospitales } \\
\text { universitarios } \\
\text { (Países Bajos) }\end{array}$ & $\begin{array}{l}\text { Evaluar el efecto de un } \\
\text { programa personalizado } \\
\text { de atención sanitaria } \\
\text { electrónica para el regreso } \\
\text { a las actividades diarias } \\
\text { después de la cirugía. }\end{array}$ & $\begin{array}{l}\text { - Grupo intervención } \\
(\mathrm{n}=173) \\
\text { (Trabajadores } n=132 \\
(76 \%)) \\
\text { - Grupo atención } \\
\text { habitual }(\mathrm{n}=171) \\
\text { (Trabajadores } \\
n=123(72 \%))\end{array}$ & $\begin{array}{l}\text { Cálculo: } \\
\text { - tiempo hasta el } \\
\text { primer día de RT. } \\
\text { - tiempo hasta el RT } \\
\text { completo. }\end{array}$ & $\begin{array}{l}\text {-Primer RT: el tiempo no } \\
\text { difirió entre ambos grupos. } \\
\text { Grupo de intervención: } \\
\text { mediana } 14 \text { días [IQR 7-21]. } \\
\text { Grupo de control: mediana } \\
13 \text { días [IQR 7-23]. } \\
\text {-RT completo: variación } \\
\text { significativa ya que la } \\
\text { expectativa en días para RT } \\
\text { del grupo de intervención } \\
\text { fue de } 14 \text { días y la de } \\
\text { control } 10 \text { días. } \\
\text { Grupo intervención: } \\
\text { mediana } 18 \text { días [IQR } \\
10-27] \text {. } \\
\text { Grupo de control: mediana } \\
19 \text { días [IQR 11-33]. }\end{array}$ \\
\hline
\end{tabular}

\begin{tabular}{|c|c|c|c|c|}
\hline $\begin{array}{l}\text { Beiwinkel et al, } \\
2017(32) \\
\text { Ensayo } \\
\text { controlado } \\
\text { aleatorio de } \\
\text { dos brazos. } \\
\text { Seguro } \\
\text { de salud } \\
\text { obligatorio } \\
\text { (Alemania) }\end{array}$ & $\begin{array}{l}\text { Evaluar la efectividad de } \\
\text { una intervención web } \\
\text { para reducir la depresión } \\
\text { leve o moderada y el } \\
\text { absentismo laboral por } \\
\text { enfermedad. }\end{array}$ & $\begin{array}{l}\text { - Grupo intervención } \\
(n=100) \\
\text { EM 47.01 (DS 10.36) } \\
\text { 66\% Mujeres } \\
\text { - Grupo atención } \\
\text { habitual ( } n=80) \\
\text { EM 48.66 (DS 11.59) } \\
71.3 \% \text { Mujeres }\end{array}$ & $\begin{array}{l}\text { Datos del registro de } \\
\text { absentismo. } \\
\text { - \% personas que } \\
\text { estuvieron ausentes } \\
\text { al menos una vez. } \\
\text { - Frecuencia de } \\
\text { ausencia (media y } \\
\text { SD). } \\
\text { - Duración de la } \\
\text { ausencia (media y } \\
\text { SD). }\end{array}$ & $\begin{array}{l}\text { Para las tres mediciones, } \\
\text { las diferencias entre los } \\
\text { grupos en la evaluación } \\
\text { posterior ( } 90 \text { días tras } \\
\text { intervención) no fueron } \\
\text { estadísticamente } \\
\text { significativas (ausencia al } \\
\text { menos una vez: } \\
F_{1,159}=.80, P=.37 \text {, } \\
\text { frecuencia de ausencia: }_{F_{1,159}=3.24, P=.07 \text {, }} \\
\text { duración de la ausencia: } \\
F_{1,159}=.02, P=.88 \text { ). }\end{array}$ \\
\hline
\end{tabular}




\begin{tabular}{|c|c|c|c|c|}
\hline $\begin{array}{l}\text { Autor, tipo } \\
\text { estudio, } \\
\text { contexto }\end{array}$ & Objetivo del estudio & Participantes & Métodos & Principales hallazgos \\
\hline
\end{tabular}

\begin{tabular}{|c|c|c|c|c|}
\hline $\begin{array}{l}\text { Ghoshchi et al, } \\
2020(33) \\
\text { Ensayo } \\
\text { controlado } \\
\text { aleatorio, } \\
\text { simple ciego, } \\
\text { de dos brazos. } \\
3 \text { hospitales } \\
\text { (Italia) }\end{array}$ & $\begin{array}{l}\text { Verificar la efectividad del } \\
\text { tratamiento tecnológico, } \\
\text { realizado con sistemas } \\
\text { ópticos (SonicHand) y } \\
\text { wearable (Riablo }{ }^{\top M} \text { ) en el } \\
\text { RT de pacientes que han } \\
\text { sufrido un ictus. }\end{array}$ & $\begin{array}{l}\text { - Grupo Intervención } \\
(n=23) \\
\text { Riablo } n=10 \\
\text { EM } 47.5 \pm 9.1 \\
\text { SoniHand } n=13 \\
\text { EM } 54.0 \pm 13.3 \\
\text { - Grupo atención } \\
\text { habitual ( } n=25) \\
\text { EM 52.5 } \pm 10.5\end{array}$ & $\begin{array}{l}\text { - Cuestionario } \\
\text { especifico de RT } \\
\text { con preguntas } \\
\text { relacionadas con } \\
\text { adaptaciones } \\
\text { organizativas o físicas } \\
\text { aplicadas al lugar de } \\
\text { trabajo } \\
\text { (personas con } \\
\text { adaptaciones en la } \\
\text { tarea, el tiempo, la } \\
\text { herramienta y } \\
\text { dispositivos de } \\
\text { ayuda). }\end{array}$ & $\begin{array}{l}\text { Los pacientes tratados con } \\
\text { rehabilitación tecnológica } \\
\text { tienen más probabilidad } \\
\text { de volver al trabajo } \\
47.8 \%(O R=9) \text { que los } \\
\text { tratados con rehabilitación } \\
\text { convencional } 36 \%(O R=6) \text {. }\end{array}$ \\
\hline
\end{tabular}

\begin{tabular}{|c|c|c|c|c|}
\hline $\begin{array}{l}\text { Blanquero et al, } \\
\text { 2020(23) } \\
\text { Ensayo } \\
\text { controlado } \\
\text { aleatorio } \\
\text { de grupos } \\
\text { paralelos. } \\
\text { Empresa: } \\
\text { Ibermutua } \\
\text { (terapia } \\
\text { ocupacional) } \\
\text { (Sevilla, } \\
\text { España) }\end{array}$ & $\begin{array}{l}\text { Verificar si la realización } \\
\text { de ejercicios guiados y } \\
\text { realizados en una pantalla } \\
\text { táctil de una tablet, } \\
\text { aceleran el regreso al } \\
\text { trabajo, reducen el uso } \\
\text { de la atención médica y } \\
\text { mejoran la recuperación } \\
\text { clínica en personas con } \\
\text { lesiones óseas y tejidos } \\
\text { blandos de la muñeca, } \\
\text { mano y dedos. }\end{array}$ & $\begin{array}{l}\text { - Grupo intervención } \\
(n=40) \\
\text { EM } 45 \text { (DS 11) } \\
68 \% \text { Hombres } \\
\text { - Grupo atención } \\
\text { habitual ( } n=34) \\
\text { EM } 42 \text { (DS 11) } \\
56 \% \text { Hombres }\end{array}$ & $\begin{array}{l}\text { - Cálculo de RT: } \\
\text { número de días del } \\
\text { calendario entre el } \\
\text { primer día de baja por } \\
\text { enfermedad y el día } \\
\text { en que el trabajador } \\
\text { fue dado de alta y } \\
\text { regresó al entorno } \\
\text { laboral. } \\
\text { - QuickDASH } \\
\text { (versión corta DASH) } \\
\text { (puntuación de } \\
\text { 0-100). }\end{array}$ & $\begin{array}{l}\text { El grupo de intervención } \\
\text { comparado con el grupo } \\
\text { control mostró mejores } \\
\text { resultados en el RT, } \\
\text { volviendo a la actividad } \\
\text { laboral antes } \\
\text { (DM - } 18 \text { días, IC del 95\% } \\
\text {-33 a -3). } \\
\text { Se demostró una leve, } \\
\text { aunque no clínicamente } \\
\text { relevante, mejora en la } \\
\text { capacidad funcional } \\
\text { percibida del grupo de } \\
\text { intervención respecto del } \\
\text { grupo control. }\end{array}$ \\
\hline $\begin{array}{l}\text { Wolf et al, } \\
2016(34) \\
\text { Ensayo } \\
\text { controlado } \\
\text { aleatorio de } \\
\text { tres brazos. } \\
\text { Hospital } \\
\text { Universitario } \\
\text { (Suecia) }\end{array}$ & $\begin{array}{l}\text { Investigar el efecto de } \\
\text { un diario de eSalud y } \\
\text { una herramienta para el } \\
\text { seguimiento de síntomas } \\
\text { en combinación con } \\
\text { atención personalizada } \\
\text { para trabajadores con } \\
\text { síndrome coronario agudo } \\
\text { (SCA). }\end{array}$ & $\begin{array}{l}\text { - Grupo atención } \\
\text { personalizada + } \\
\text { eSalud ( } n=37) \\
\text { EM } 59.8 \text { (DS 10.1) } \\
\text { 19\% Mujeres } \\
\text { - Grupo atención } \\
\text { personalizada sin } \\
\text { eSalud ( } n=57 \text { ) } \\
\text { EM 60.9 (DS 8.7) } \\
\text { 28.1\% Mujeres } \\
\text { - Trabajadores grupo } \\
\text { atención habitual } \\
\text { ( } n=105 \text { ) } \\
\text { EM 61.3 (DS 8.9) } \\
\text { 30.5\% Mujeres }\end{array}$ & $\begin{array}{l}\text { - Cálculo del \% } \\
\text { de personas que } \\
\text { retornaron al trabajo. }\end{array}$ & $\begin{array}{l}\text { La proporción de pacientes } \\
\text { que regresaron al trabajo } \\
\text { fue similar entre los grupos } \\
\text { a los } 6 \text { meses (atención } \\
\text { personalizada + eSalud } \\
\text { 30/34, 88\%; atención } \\
\text { personalizada sin eSalud } \\
47 / 53,89 \% \text {; control 89/98, } \\
91 \% \text { ). }\end{array}$ \\
\hline
\end{tabular}




\begin{tabular}{|c|c|c|c|c|}
\hline $\begin{array}{l}\text { Autor, tipo } \\
\text { estudio, } \\
\text { contexto }\end{array}$ & Objetivo del estudio & Participantes & Métodos & Principales hallazgos \\
\hline
\end{tabular}

\begin{tabular}{|c|c|c|c|c|}
\hline $\begin{array}{l}\text { de Jong et al, } \\
2020^{(35)} \\
\text { Ensayo } \\
\text { controlado } \\
\text { aleatorio. } \\
4 \text { centros } \\
\text { hospitalarios } \\
\text { (Países bajos) }\end{array}$ & $\begin{array}{l}\text { Evaluar desde una } \\
\text { perspectiva social, la } \\
\text { rentabilidad del uso de } \\
\text { telemedicina con la } \\
\text { herramienta mylBDcoach } \\
\text { en comparación con } \\
\text { la atención estándar } \\
\text { trabajadores con } \\
\text { enfermedad intestinal } \\
\text { inflamatoria(Crohn y } \\
\text { Colitis ulcerosa). }\end{array}$ & $\begin{array}{l}\text { - Grupo intervención } \\
(n=465) \\
\text { EM 44 (DS 14.1) } \\
58.3 \% \text { Mujeres } \\
\text { - Grupo control } \\
\text { (n=444) } \\
\text { EM 44.1 (DS 14.2) } \\
\text { 59.5\% Mujeres }\end{array}$ & $\begin{array}{l}\text { - Cálculo de días de } \\
\text { ausencia por IT. } \\
\text { - WPAI }\end{array}$ & $\begin{array}{l}\text { La media de los costes por } \\
\text { pérdida de productividad } \\
\text { debida al absentismo fue } \\
\text { de } 1886 € \text { en el grupo de } \\
\text { intervención y } 2058 € \text { en } \\
\text { el grupo de control. Esto } \\
\text { resultó en un ahorro medio } \\
\text { anual de } 172 € \text { por paciente } \\
\text { (95\% intervalo de confianza } \\
\text { [IC], -848€ a } 1192 € \text { ) } \\
\text { favoreciendo el uso de } \\
\text { telemedicina. }\end{array}$ \\
\hline
\end{tabular}

\begin{tabular}{ll}
\hline Schaetz et al, & Evaluar el impacto de un \\
$2020{ }^{(36)}$ & programa de manejo de \\
Estudio & migraña que incorpora \\
descriptivo de & un módulo de eSalud \\
cohortes. & ofrecido como un servicio \\
Compañia & complementario por una \\
Novartis & empresa dentro de su \\
Pharma AG, & programa de bienestar \\
(Suiza) & corporativo.
\end{tabular}

$\begin{array}{ll}\text { - Trabajadores y } & \text { - Análisis estadístico } \\ \text { familiares }(n=141) & \text { de } \\ \text { EM 41.0 (DS 9) } & \text { absentismo y } \\ 73.0 \% \text { Mujeres } & \text { presentismo. } \\ & \text { - ROI } \\ & \text { - MIDAS }\end{array}$

El presentismo disminuyó un 64\% (reducción media [DE]: 5,3 [8,0], IC del 95\%: 3,5-7,1). El absentismo disminuyó desde el inicio en un 63\% (reducción media [DE]: 4.5 [8.2], IC del 95\%: 2.6-6.5) ROI positivo de 490\% (95\% Cl: $410 \%-570 \%$ ).

\begin{tabular}{ll}
\hline Mochari- & Evaluar los resultados \\
Greenberger et & clínicos y laborales \\
al, 2020(37) & de un programa de terapia \\
Estudio & conductual virtual basado \\
descriptivo & en evidencia \\
de cohortes & para personas con dolor \\
retrospectivo. & y problemas de salud \\
(Nueva York, & conductual. \\
EEUU) &
\end{tabular}

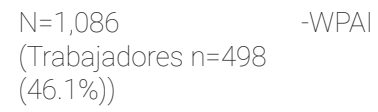

\begin{tabular}{ll}
\hline Anderson et al, & Determinar si la \\
$2019(38)$ & incorporación de un \\
Estudio de & programa digital en el \\
cohortes & protocolo de rehabilitación \\
prospectivo. & estándar en cirugías de \\
(San Francisco, & reparación del manguito \\
EEUU) & de rotadores \\
& tendría mejores \\
& resultados en el tiempo de \\
& RT trabajo y reducción de \\
& reintervenciones que con \\
& la atención habitual.
\end{tabular}

$\begin{array}{ll}\text { Pacientes en IT por } & \text { Cálculo estadístico de } \\ \text { contingencia laboral } & \text { la media de días hasta } \\ \text { sometidos a cirugía } & \text { el RT completo o con } \\ \text { de reparación del } & \text { limitaciones. } \\ \text { manguito rotador. } & \\ \text {-Grupo intervención: } & \\ \text { ( } n=23) & \\ 73.8 \% \text { Mujeres } & \\ \text {-Grupo atención } & \\ \text { habitual: }(n=25) & \\ 44 \% \text { Mujeres } & \end{array}$

-El intervalo de tiempo

medio

para RT con limitaciones /

adaptaciones fue

$17,0 \pm 7,6$ semanas para

el grupo intervención en

comparación

con 26,7 \pm 17,6 semanas

para el grupo control

con una diferencia

estadísticamente

significativa

$(P=0,017)$

-En cuanto al RT completo

los pacientes que utilizaron

eSalud volvieron antes, 28,1

\pm 9,0 semanas frente a 35,2

$\pm 18,0$

semanas para los del grupo

control $(p=0,091)$. 


\begin{tabular}{|c|c|c|c|c|}
\hline $\begin{array}{l}\text { Autor, tipo } \\
\text { estudio, } \\
\text { contexto }\end{array}$ & Objetivo del estudio & Participantes & Métodos & Principales hallazgos \\
\hline
\end{tabular}

\begin{tabular}{|c|c|c|c|}
\hline $\begin{array}{l}\text { Beaton et al, } \\
2020(40) \\
\text { Estudio } \\
\text { exploratorio } \\
\text { cualitativo. } \\
\text { (Canadá) }\end{array}$ & $\begin{array}{l}\text { Diseñar un recurso } \\
\text { innovativo educativo } \\
\text { digital para guiar } \\
\text { el conocimiento, la } \\
\text { recuperación y el } \\
\text { retorno al trabajo de } \\
\text { los trabajadores con } \\
\text { conmociones cerebrales. }\end{array}$ & $\begin{array}{l}\text { - Trabajadores } \\
(n=31) \\
70.9 \% \text { Mujeres } \\
\text { - Profesionales } \\
(n=16)\end{array}$ & $\begin{array}{l}\text { - Grupo focal de } \\
\text { discusión } \\
\text { (entrevista) } \\
\text { (14-150 min) } \\
\text { Grabada } \\
\text { - Análisis temático } \\
\text { cualitativo de las } \\
\text { transcripciones de los } \\
\text { audios. }\end{array}$ \\
\hline
\end{tabular}

Se identificaron 20 recomendaciones para mejorar la herramienta digital y adecuarla a las necesidades físicas y cognitivas de trabajadores que han padecido una conmoción cerebral con objetivo de mejorar la gestión del retorno al trabajo

\begin{tabular}{lll}
\hline Karpman et al, & Evaluar los datos de los & $\mathrm{N}=46$ \\
$2019(43)$ & dispositivos ActiGraph & EM 23.7 (DS 3.7) \\
Estudio & durante la & $54.3 \%$ Hombres \\
analítico & evaluación de la & \\
transversal. & capacidad funcional & \\
(Canadá) & (FCE) de un grupo de & \\
& trabajadores.
\end{tabular}

$\begin{array}{ll}\text { - FCE (5 ítems) : } & \text { Se mostró únicamente } \\ \text { cálculo del potencial } & \text { una relación moderada } \\ \text { para realizar tareas } & \text { entre el uso del dispositivo } \\ \text { relacionadas con el } & \text { ActiGraph y los resultados } \\ \text { trabajo (levantar, } & \text { de máximo rendimiento de } \\ \text { cargar, etc.) y si } & \text { los trabajadores durante la } \\ \text { una persona se } & \text { evaluación de capacidad } \\ \text { ha recuperado } & \text { funcional (FCE) en el RT. } \\ \text { adecuadamente de } & \\ \text { una lesión para el RT. } & \end{array}$

\begin{tabular}{|c|c|c|c|c|}
\hline $\begin{array}{l}\text { Engdahl et al, } \\
2020^{(39)} \\
\text { Estudio } \\
\text { cualitativo } \\
\text { descriptivo } \\
\text { (Suecia) }\end{array}$ & $\begin{array}{l}\text { Examinar las } \\
\text { perspectivas de tres } \\
\text { grupos interesados sobre } \\
\text { el papel y la legitimidad } \\
\text { de una solución de RT } \\
\text { digital llamada mWorks } \\
\text { para trabajadores con } \\
\text { trastornos mentales } \\
\text { comunes que están en } \\
\text { situación de IT. }\end{array}$ & $\begin{array}{l}\text { - Personal } \\
\text { administración }(n=8) \\
\text { EM } 53 \\
\text { - Profesionales } \\
\text { gestión RT }(n=20) \\
\text { EM } 42.4 \\
\text { - Trabajadores } \\
(n=18) \\
\text { EM } 38.3\end{array}$ & $\begin{array}{l}\text { - Entrevistas } \\
\text { individuales semi } \\
\text { estructuradas } \\
\text { (30-45 min). } \\
\text { - Grupos focales } \\
\text { (45-60 min) } \\
\text { (4 items experiencia } \\
\text { con eSalud, } \\
\text { perspectivas de } \\
\text { la herramienta } \\
\text { mWorks, obstáculos } \\
\text { implementación y } \\
\text { factores de éxito). }\end{array}$ & $\begin{array}{l}\text { La legitimidad de la } \\
\text { herramienta digital fue alta } \\
\text { entre todos los grupos } \\
\text { ya que se percibió que } \\
\text { permitía a los usuarios } \\
\text { tomar el control de } \\
\text { su proceso de RT. La } \\
\text { herramienta mostro un } \\
\text { gran potencial como } \\
\text { producto de apoyo y } \\
\text { empoderamiento para la } \\
\text { toma de decisiones en el } \\
\text { RT. }\end{array}$ \\
\hline $\begin{array}{l}\text { Gross et al, } \\
2020(41) \\
\text { Estudio } \\
\text { cualitativo } \\
\text { descriptivo } \\
\text { Transversal. } \\
\text { (Canadá) }\end{array}$ & $\begin{array}{l}\text { Evaluar los resultados } \\
\text { del programa de } \\
\text { rehabilitación FIT en } \\
\text { trabajadores con lesiones } \\
\text { musculoesqueléticas vía } \\
\text { telesalud. }\end{array}$ & $\begin{array}{l}\text { - Población } \\
\text { trabajadora } \\
\text { grupo en remoto } \\
\text { ( } n=1223 \text { ) } \\
\text { EM 45.8 (DS 12.5) } \\
60.3 \% \text { Hombres } \\
\text { - Grupo atención } \\
\text { habitual ( } n=3293 \text { ) } \\
\text { EM 45.9 (DS 12.5) } \\
59.3 \% \text { Hombres }\end{array}$ & $\begin{array}{l}\text { - Recomendaciones } \\
\text { en la evaluación de RT } \\
\text { (con o sin adaptación } \\
\text { al puesto). } \\
\text { - Naturaleza de las } \\
\text { recomendaciones de } \\
\text { RT (en las tareas, las } \\
\text { horas o ambas). } \\
\text { - Limitaciones para } \\
\text { realizar el trabajo } \\
\text { (temporales o } \\
\text { permanentes). } \\
\text { - No RT. }\end{array}$ & $\begin{array}{l}\text { Las evaluaciones de RT } \\
\text { realizadas con tecnología } \\
\text { remota fueron exitosas, } \\
\text { pero se manejaron con } \\
\text { más cautela que en el } \\
\text { grupo control. } \\
\text { Los profesionales que } \\
\text { realizaron las evaluaciones } \\
\text { recomendaron más } \\
\text { limitaciones para } \\
\text { desarrollar el trabajo en } \\
\text { el RT, siendo un gran } \\
\text { porcentaje de ellas } \\
\text { permanentes. }\end{array}$ \\
\hline
\end{tabular}




\begin{tabular}{|c|c|c|c|c|}
\hline $\begin{array}{l}\text { Autor, tipo } \\
\text { estudio, } \\
\text { contexto }\end{array}$ & Objetivo del estudio & Participantes & Métodos & Principales hallazgos \\
\hline $\begin{array}{l}\text { Noben et al, } \\
(2015)^{(44)} \\
\text { Estudio de } \\
\text { evaluación } \\
\text { económica. } \\
\text { (Países bajos) }\end{array}$ & $\begin{array}{l}\text { Realizar un estudio piloto } \\
\text { para la implantación } \\
\text { de intervenciones de } \\
\text { asistencia eSalud que } \\
\text { enseñan a trabajadores } \\
\text { con discapacidades } \\
\text { laborales las habilidades } \\
\text { para regresar y / o } \\
\text { permanecer en el trabajo. }\end{array}$ & $\begin{array}{l}\text { - Grupo intervención } \\
(n=29) \\
\text { EM } 51 \text { (DS 8) } \\
76 \% \text { Mujeres } \\
\text { - Grupo atención } \\
\text { habitual } \\
(n=34) \\
\text { EM 53 (DS 7.9) } \\
53 \% \text { Mujeres }\end{array}$ & $\begin{array}{l}\text { - WAl } \\
\text { - Costes de } \\
\text { productividad } \\
\text { (absentismo y } \\
\text { presentismo según } \\
\text { el Dutch Manual for } \\
\text { costing in economic } \\
\text { evaluations). }\end{array}$ & $\begin{array}{l}\text { Los resultados no } \\
\text { mostraron mejoras } \\
\text { significativas comparando } \\
\text { las medias de costes } \\
\text { con las estimadas. Se } \\
\text { deberán realizar cambios } \\
\text { en la aplicación antes de } \\
\text { extender su implantación. }\end{array}$ \\
\hline $\begin{array}{l}\text { Collins et al, } \\
2020^{(42)} \\
\text { Estudio piloto } \\
\text { de evaluación. } \\
\text { (Randwick, } \\
\text { Australia) }\end{array}$ & $\begin{array}{l}\text { Evaluar la usabilidad, la } \\
\text { viabilidad, la aceptabilidad } \\
\text { y los efectos preliminares } \\
\text { de una intervención } \\
\text { basada en una aplicación } \\
\text { diseñada para reducir } \\
\text { la depresión y mejorar } \\
\text { la salud mental en la } \\
\text { población laboral en } \\
\text { general. }\end{array}$ & $\begin{array}{l}\text { Trabajadores con } \\
\text { alto nivel de estrés } \\
(n=81) \\
\text { EM } 38.96 \text { (DS 10.34) } \\
67.9 \% \text { Mujeres }\end{array}$ & $\begin{array}{l}\text { - HPQ (pregunta } \\
\text { "presentismo } \\
\text { absoluto"). } \\
\text { - Absentismo a corto } \\
\text { plazo: número de días } \\
\text { por enfermedad en } \\
\text { los últimos } 28 \text { días. } \\
\text { Pregunta adicional } \\
\text { = días ausentes por } \\
\text { motivos de salud } \\
\text { mental. } \\
\text { - Escala BRS de } \\
\text { resiliencia. }\end{array}$ & $\begin{array}{l}\text { No se informaron } \\
\text { cambios significativos } \\
\text { en el desempeño laboral } \\
\left(t_{60}=-0.97 ; p=0.338\right) \\
\text { y el absentismo } \\
\left(t_{60}=-0.18 ; p=0.861\right) \text {. } \\
\text { Se evidenció una mejora en } \\
\text { la resiliencia ( } t_{60}=-3.89 ; p< \\
0.001) \text {. }\end{array}$ \\
\hline
\end{tabular}

BRS: Brief Resilience Scale. Escala rápida de 6 ítems con 5 opciones de respuesta que van de muy de acuerdo (5) a muy en desacuerdo (1); CD-RISC: Connor-Davidson Resilience Scale. Escala de resiliencia 10 ítems (puntuación 0-40 corresponden a mayor resiliencia); Cox: es un método de regresión para los datos de supervivencia, proporciona una estimación de la razón de riesgo y su intervalo de confianza. La razón de riesgo es una estimación de la razón de la tasa de riesgo en el grupo tratado frente al grupo de control; DASH: Disabilities of the Arm, Shoulder and Hand questionnaire. Es un cuestionario de autoinforme de 30 ítems que mide la función física y los síntomas en personas con trastornos musculoesqueléticos de las extremidades superiores; DM: diferencia de medias; DS: desviación estándar; EM: edad media; FCE: Functional capacity evaluation. Cálculo del potencial para realizar tareas relacionadas con el trabajo (levantar, cargar, etc.) e identificar si una persona se ha recuperado adecuadamente de una lesión para el RT); HPQ: Health and work Performance Questionnaire. Cuestionario con13 preguntas relativas al trabajo y 8 sobre datos demográficos; HR: Hazard ratio. Razón de las tasas de riesgo correspondientes a las condiciones descritas por dos niveles de una variable explicativa; iPCQ: iMTA Productivity Costs Questionnaire. Incluye tres módulos que miden las pérdidas de productividad del trabajo remunerado debido a absentismo, presentismo así como para trabajos no remunerados y pérdidas de productividad; iSMI Internet-based Stress Management Intervention. Escala numérica para el manejo del estrés basada en Internet; MIDAS: Migraine Disability Assessment questionnaire. Cuestionario de 5 preguntas (puntuación 0 a 90) que se utiliza para clasificar a los pacientes en los grados de discapacidad de I a IV; PRODISQ: Productivity and Disease Questionnaire. Cuestionario modular que cubre todos los aspectos relevantes de la relación entre salud y productividad, incluida la ausencia del trabajo; Prueba F (F): término aplicado a cualquier test que utiliza distribución F; REQ-PD: Psychological Detachment Subscale of the Recovery Experience Questionnaire. Rol: Return on investment : valor inicial de la inversión del valor final de la inversión (que es igual al rendimiento neto), luego dividiendo este nuevo número (el rendimiento neto) por el costo de la inversión y, finalmente, multiplicándolo por 100; RT: retorno al Trabajo; RT parcial reanudar el trabajo con el $50 \%$ de los ingresos; RT completo: reanudar el trabajo con el 100\% de los ingresos; RT completo**: la reanudación del trabajo propio u otro trabajo con iguales ingresos (parcial o totalmente), durante al menos 4 semanas sin que se repita parcial o totalmente) la baja por enfermedad; SPS-6: Stanford Presenteeism Scale. Cuestionario de autoinforme de 6 ítems. Mide el impacto de la capacidad percibida de un trabajador para concentrarse en las tareas laborales a pesar de las distracciones de los problemas de salud y el dolor a través de escala Likert de 5 items (1: muy de acuerdo y 5: muy en desacuerdo); TIC-P: Treatment Inventory of Costs in patients with Psychiatric disorders. Consta de 2 partes. La primera, 14 preguntas estructuradas sobre el volumen de consumo médico. La segunda parte trata de las pérdidas de productividad; UWES: Utrecht Work Engagement Scale. 16 ítems para evaluar el compromiso laboral; WAI: Work Ability Index. 7 ítems ( 2, 3 y 7 están compuestos por los sub ítems 2, 14 y 3, respectivamente). Una puntuación más alta indica una mejor capacidad de trabajo; WPAI: Work Productivity and Activity Impairment. Es un cuestionario de autoinforme de 30 ítems que mide la función física y los sintomas en personas con trastornos musculoesqueléticos de las extremidades superiores. 


\section{Retorno al trabajo}

Entre el total de estudios seleccionados, 9 mostraron datos sobre el RT siendo muy diversas las herramientas utilizadas para llevar a cabo la medición del RT. Se encontró que 8 de los 9 estudios mostraron un efecto positivo del uso de la salud digital en el RT.

Según Volker et al(28) en el primer RT, se mostró una diferencia de 27 días entre el grupo de intervención (media 99.0, DS 78.8 días) y el grupo de cuidado tradicional (media 72.5, DS 71.1 días). Sin embargo, no hubo diferencia significativa en los tiempos de RT completo entre ambos grupos. Resultados cruzados se obtuvieron en Van der Meij et al(22) que registró variaciones en el RT completo y ninguna significativa en el primer RT en una muestra de trabajadores sometidos a cirugía abdominal.

Bouwsma et al ${ }^{(31)}$, demostró que el uso de un programa de atención basado en Internet en trabajadoras sometidas a histeroscopia y laparoscopia quirúrgica por enfermedad benigna, mejoró las tasas de RT. Se tuvieron en cuenta los tiempos de IT esperados para cada tipo de cirugía, por lo que, al igual que en Volker et al(28), se aplicó el modelo de Cox para comparar grupos heterogéneos.

Resultados positivos de la herramienta digital también se mostraron en el estudio de Blanquero et al ${ }^{(23)}$. Se evaluó el efecto de una tablet táctil (ReHand) para acelerar el RT en personas con lesiones de muñeca, mano y dedos, encontrando que los participantes del grupo de intervención retornaron al trabajo 18 días antes que el grupo de cuidado tradicional.

Para Anderson et al(38) el RT con adaptaciones se produjo a las $17 \pm 7.6$ semanas en comparación con el grupo de cuidado tradicional que fue de $26.7 \pm 17.6$ semanas $(P=0.017)$. Se observó también una mejora del RT completo de $21.1 \pm 9$ semanas en el grupo de intervención respecto del grupo de control $35.2 \pm 18$ semanas $(P=0.091)$.

Las herramientas digitales (Riablo y SoniHand) utilizadas en el campo de la neurorrehabilitación, según Ghoshchi et al(33), mostraron también resultados positivos. La posibilidad de retorno al trabajo fue del $47.8 \%(O R=9 ; p=0.027)$ en el grupo que utilizó las herramientas digitales respecto a un $36 \%(\mathrm{OR}=6 ; \mathrm{p}=0.05)$ en el grupo control.

En el estudio de Karpman et al(43), se utilizó la evaluación de la capacidad funcional (FCE), considerada de gran ayuda para facilitar el proceso de $\mathrm{RT}^{(45)}$ al medir la capacidad para realizar tareas laborales a través de una serie de test, en trabajadores en situación de baja laboral.

Engdahl et al ${ }^{(39)}$ a través de entrevistas semiestructuradas y grupos focales de tres grupos, trabajadores, profesionales gestores del RT y personal de administración, demostró la legitimidad y el potencial de la herramienta digital (mWorks) como herramienta apoyo para el RT.

No se recogieron resultados favorables significativos para la salud digital en $1^{(34)}$ de los 9 estudios. Los autores expresaron que la intervención de salud digital no 
fue considerada por la mayoría de pacientes como herramienta de apoyo debido a que, como sugieren algunos estudios cualitativos, los pacientes con fallo cardiaco congestivo le dan mayor importancia a la atención presencial por parte del profesional(46).

\section{Absentismo}

Se encontraron 13 estudios que mostraron resultados sobre absentismo en los que se utilizaron también una gran diversidad de herramientas para medir los datos, de los cuales 5 demostraron un efecto beneficioso de la salud digital en el absentismo.

Lokman et al(25), en una muestra de trabajadores en situación de IT por trastornos mentales, la intervención digital demostró reducir ambos, absentismo y presentismo con un ROI de 11 euros por euro invertido. Los costes acumulados por absentismo a los 12 meses fueron de $16.445 €$ en el grupo de intervención y de 20.736 $€$ en el de control.

Autores como de Jong et al(35) y Mochari-Greenberger et al ${ }^{(37)}$, obtuvieron resultados de absentismo a partir del cuestionario WPAI (Work Productivity and Activity Impairment) utilizado como instrumento de medida para determinar las consecuencias del dolor en la productividad laboral. Según de Jong et al ${ }^{(35)}$ las horas de pérdida de productividad fueron de 54.3 horas anuales (DS 232.6) en el grupo que utilizó la herramienta digital y de 59.1 (218.4) para el grupo de control. Mochari-Greenberger et al( ${ }^{(37)}$ evidenció una mejora del absentismo del 39\% para el grupo de eSalud.

Hutting et al(29) y Shaetz et al(36) recogieron los datos a partir del cálculo de los días de ausencia. Este último ${ }^{(36)}$ incorporó un enfoque digital en un programa dirigido a trabajadores diagnosticados con migraña, demostrando una disminución en el absentismo en el mes 9 (reducción media [DE]: 4.5 [8.2], IC del 95\%: 2.6-6.5). Se mostró un ROI positivo de 490\% (95\% Cl: 410\%-570\%).

Entre el total, 8 estudios $(21,26,27,28,30,32,42,44)$, mostraron que la salud digital no proporcionaba datos beneficiosos significativos en relación al absentismo, sin embargo cabe señalar que no fue negativo en ninguno de los casos.

\section{Presentismo}

En 10 estudios se observaron datos sobre presentismo, con resultados positivos para la eSalud en 5 de ellos. Al igual que en el absentismo y retorno al trabajo, se utilizó una gran variedad de métodos de medición.

Deady et al(24), a través del Health and work Performance Questionnaire (HPQ) obtuvo una diferencia de medias entre los grupos a estudio de 1.73 (IC 95\% 0.602.86) ( $p=0.003)$, siendo positivo para el grupo de intervención. Para evaluar los costes por presentismo Lokman et al ${ }^{(25)}$ calculó el número de días trabajados por un coeficiente de ineficacia reportado por los propios trabajadores (de 0: efectivo 
a 1: totalmente inefectivo). A los 3 y 6 meses se observó un empeoramiento en el coste respecto al grupo de control, que según el autor fue debido a la mejoría en el absentismo. Sin embargo, se mostraron resultados positivos a largo plazo (a los doce meses: $493 €$ en el grupo de control y $325 €$ en el de intervención). Ebert et al ${ }^{(30)}$, utilizó el mTrimbos and Institute of Medical Technology Assessment Cost Questionnaire for Psychiatry (TiC-P-G) para medir el presentismo, mostrando mejoras en el tercer trimestre a favor del grupo de intervención con una media de pérdida de días de 7.2 (DS 9.6), mientras que en el grupo de control fueron de 10.5 días (DS 12.2). Los datos que obtuvo Schaetz et al(36), en un estudio de cohortes, indicaron que la media de días perdidos pasó de 8.3 (DS 7.5) a 3.0 (DS 4.5) a los 9 meses.

Mochari Greenberger et al(37), observó una mejora del presentismo del 28\% en la puntuación del cuestionario WPAl realizado al finalizar el estudio (8 semanas).

En 5 de los estudios $(26,27,29,42,44)$, no se mostraron datos relevantes en relación al presentismo aunque en ningún caso negativos.

\section{Otros factores}

Durante el proceso de análisis de los estudios, se observó que algunos autores mostraron resultados sobre otros factores relacionados con la salud en el trabajo. La resiliencia, el desapego psicológico al trabajo y el compromiso laboral se consideraron influyentes sobre los índices de absentismo y el retorno a la actividad laboral ${ }^{(24,30,42) \text {. }}$

\section{Herramientas digitales}

La herramienta mayormente utilizada fue la aplicación web, seguida de las aplicaciones específicas para móviles y tablets. En 6 de los estudios( ${ }^{(21,22,30,34,37,44)}$ se utilizó una combinación de dos o varias herramientas digitales. Durante la revisión, se pudo observar la evolución del término de salud digital o eSalud, el cual hace algunos años hacía referencia, principalmente, a la digitalización de los registros médicos y el intercambio de datos entre profesionales. Progresivamente otros dispositivos tecnológicos de última generación como aplicaciones móviles, wearables o la videoconferencia están siendo implantadas no solo para intervenciones de promoción de la salud y tratamiento, sino que adquieren cada vez mayor presencia en programas de rehabilitación, para impulsar la autogestión de la salud e incluso, en intervenciones más gestoras como en programas de absentismo y reinserción laboral.

\section{Conclusiones y perspectivas}

El objetivo de esta revisión fue analizar el impacto de las herramientas de salud digital en los resultados de absentismo y retorno al trabajo de la población activa. Para ello, se seleccionaron veinticuatro estudios de los cuales diez, además apor- 
taron datos sobre presentismo y otros factores influyentes en el absentismo y el retorno a la actividad laboral, como fueron la resiliencia, el compromiso laboral y el desapego psicológico al trabajo.

La presente revisión ofrece una serie de limitaciones que hay que considerar em la interpretación de los resultados. En primer lugar, los datos sobre absentismo y RT son mostrados en la mayor parte de los estudios como resultados secundarios. Además, 10 de los 24 estudios se realizaron en Países Bajos, por lo que los resultados obtenidos podrían verse influenciados por los factores socio laborales de este país. En segundo lugar, no se pudieron establecer comparaciones cuantitativas de los datos extraídos debido a la variabilidad de las mediciones realizadas por parte de los autores y por ende, a la heterogeneidad de resultados, por lo se utilizó un método de cálculo que permitiera la comparación entre estudios para poder dar respuesta a los objetivos específicos por lo que se decidió tratar los datos recogidos, individualmente. En tercer lugar, los estudios utilizaron diferentes tipos de herramientas digitales (plataformas web, aplicaciones móviles, wearables, entre otros.) dirigidas a trabajadores con patologías diversas. Por este motivo, tampoco pudo realizarse una comparación exhaustiva entre los resultados obtenidos en conjunto. A pesar de las limitaciones mencionadas, cabe resaltar que en ninguno de los 24 estudios se observó que el uso de las herramientas digitales ocasionara un perjuicio.

Los estudios demostraron, mayoritariamente, resultados positivos, evidenciándose el impacto favorable del uso de la salud digital en el la gestión del retorno al trabajo, el absentismo y el presentismo. Sin embargo, cabe señalar que en algunos de los casos la mejora no resultó significativa respecto al cuidado habitual.

El retorno al trabajo (parcial o completo) presentó una media de $32.24 \%$ de mejora en favor de la eSalud. El porcentaje obtenido en relación con el absentismo fue menor, aunque igualmente positivo, mostrando una media del $11.58 \%$. El presentismo fue el factor que evidenció el mayor porcentaje de mejora, demostrando una media de $37.77 \%$. Los datos se extrajeron de los estudios seleccionados que aportaron datos cuantitativos, calculando el porcentaje de mejora en ambos, el grupo de intervención y control. En los casos en los que no se hubo diferencias significativas, el porcentaje de mejora se consideró del $0 \%$.

La resiliencia, el desapego psicológico al trabajo y el compromiso laboral fueron considerados como factores influyentes en los índices de absentismo y de retorno al trabajo por Ebert et al ${ }^{(30)}$, Deady et al ${ }^{(24)}$ y Collins et al ${ }^{(42)}$. Como dato adicional se encontró que algunos de los autores ${ }^{(25,27,29,31)}$ hicieron referencia a una mejora de la autogestión y la autoeficacia en los pacientes que utilizaron herramientas de eSalud.

Estudios anteriores pusieron de manifiesto la relación entre la autoeficacia y la reincorporación laboral. Nieuwenhuijsen et al (2013)(47), definió la autoeficacia como factor predictor del retorno real al trabajo. Volker et al (2014)(48) consideró a la autoeficacia como predictor significativo de la reintegración al trabajo en los trabajadores con absentismo de larga duración. En cuanto a la autogestión, Bolier 
et al (2013)(49), subrayó el beneficio de las intervenciones de salud electrónica en manejo de enfermedades crónicas.

Aunque esta revisión muestra la influencia positiva de la eSalud en la gestión del absentismo y retorno al trabajo, se precisan, sin embargo, futuras investigaciones en este área de la salud laboral que aporten datos homogéneos, que faciliten la comparación entre resultados.

Ante la transformación que está sufriendo el empleo y las condiciones de trabajo como consecuencia de las tecnologías emergentes es importante que los enfermeros del trabajo, como gestores de la salud laboral, deban conocer el alcance que dichas tecnologías ofrecen al trabajador y la empresa.

El presente trabajo analiza el efecto de la salud digital en intervenciones que involucran exclusivamente el área gestora de la salud. En la literatura se encuentran pocos estudios que estén relacionados con las tecnologías digitales que muestren datos sobre absentismo y retorno al trabajo como resultados primarios. Además la falta de exhaustividad y coherencia en el uso de herramientas para medir el retorno al trabajo y el absentismo supone un impedimento para poder establecer un análisis comparativo, circunstancia que ha dificultado la realización de esta revisión sistemática y que incluso, podría afectar al avance de su investigación.

A modo de conclusiones, podría decirse que:

- La salud digital mejora los resultados de la gestión del absentismo laboral y el retorno al trabajo en comparación con los métodos habituales de gestión de la salud.

- El uso de herramientas digitales favorecen el retorno a la actividad laboral, con o sin adaptaciones del puesto, de aquellos trabajadores que se encuentran en situación de incapacidad temporal por causa de enfermedad.

- Por ende, la salud digital facilita la reducción de las cifras de absentismo laboral, debido a la relación que existe entre ambos términos, sin embargo, la mejora es menos significativa en las intervenciones enfocadas a la gestión del absentismo que las dirigidas al retorno al trabajo.

- La salud digital contribuye positivamente en mayor o menor medida sobre el presentismo laboral, en especial en aquellos trabajadores que padecen enfermedades crónicas o de larga duración y como consecuencia de la influencia positiva de las herramientas digitales sobre la autoeficacia y la autogestión.

- La resiliencia, el desapego psicológico al trabajo y el compromiso laboral son considerados factores influyentes sobre los resultados de absentismo y de retorno a la actividad laboral.

- Entre las herramientas más utilizadas por los diferentes autores se encuentran las plataformas web seguidas de las aplicaciones para móviles y tablets.

- Esta revisión demuestra el potencial de las intervenciones de salud digital para contribuir positivamente a disminuir el impacto personal, social y económico de las enfermedades crónicas y de larga duración. 
- Se precisan, sin embargo, futuras investigaciones que aporten nuevos datos:

1. En cuanto al retorno al trabajo, el absentismo y el presentismo, sería de gran utilidad para los gestores, la utilización en los estudios de un método de medición estándar que permitiera realizar un marco comparativo de resultados.

2. Igualmente beneficioso sería que se llevaran a cabo estudios que compararan el efecto de la aplicación de diferentes tipos de herramientas digitales sobre una patología en concreto, de manera que se identificasen las más beneficiosas para la gestión de determinadas enfermedades.

3. Adicionalmente, se precisarían estudios que fueran realizados en el lugar de trabajo que permitieran un mayor periodo de seguimiento y así evaluar el impacto a más largo plazo sobre los factores analizados.

\section{Financiación}

El presente trabajo no esta financiado.

\section{Conflicto de intereses}

No existe ningún conflicto de intereses.

\section{Bibliografía}

1. Page MJ, McKenzie JE, Bossuyt PM, Boutron I, Hoffmann TC, Mulrow CD, et al. The PRISMA 2020 statement: an updated guideline for reporting systematic reviews. BMJ. 2021;372:n71 Disponible en: http://prisma-statement.org/documents/PRISMA_2020_checklist.pdf

2. Joanna Briggs Institute. Critical appraisal tools [Internet]. [consultado 14 Ago 2021]. Disponible en: https://jbi.global/critical-appraisal-tools

3. Golinelli D, Boetto E, Carullo G, Nuzzolese AG, Landini MP, Fantini MP. Adoption of digital technologies in health care during the COVID-19 pandemic: Systematic review of early scientific literature. JMIR Publications [Internet]. 1 Nov 2020 [consultado 28 Mar 2021];22(11):e22280. Disponible en: https://www.ncbi.nlm.nih. gov/pmc/articles/PMC7652596/

4. Burgel BJ, Childre F. The Occupational Health Nurse as the Trusted Clinician in the 21st Century. Workplace Health \& Safety [Internet]. Abr 2019 [consultado 28 Mar 2021];60(4):143-50. Disponible en: https://journals.sagepub.com/doi/10.117 7/216507991206000402?url_ver=Z39.88-2003\&rfr_id=ori:rid:crossref.org

5. Romero Saldaña M, Moreno Pimentel AG, Santos Posada A. Occupational Health Nursing: Competence and experience to achieve the safety, health and well-being of the working population. Enfermería Clínica [Internet]. 1 Nov 2019 [consultado 28 Mar 2021];29(6):376-80. Disponible en: https://www.sciencedirect.com/ science/article/abs/pii/S1130862119303705?via\%3Dihub 
6. González González, César; Wong R. Impacto de la salud: análisis longitudinal del empleo en edad media y avanzada en México. 2014 [consultado 28 Mar 2021];20(81). Disponible en: http://www.scielo.org.mx/scielo.php?script=sci_arttext\&pid=S1405-74252014000300005

7. Nazarov S, Manuwald U, Leonardi M, Silvaggi F, Foucaud J, Lamore K, et al. Chronic diseases and employment: Which interventions support the maintenance of work and return to work among workers with chronic illnesses? a systematic review [Internet]. International Journal of Environmental Research and Public Health. 2019;16 [consultado 28 Mar 2021]. Disponible en: https://www.ncbi.nlm.nih.gov/ pmc/articles/PMC6572561/

8. Solé Gómez, Ma Dolores; Cobos Sanchiz D. Mantenimiento y vuelta al trabajo: procedimiento [Internet]. 2018 [consultado 28 Mar 2021]. Disponible en: https:// www.insst.es/documents/94886/566858/ntp_1116w.pdf/c851d358-0fd7-4f72a273-9682d21c9760

9. Blasco de Luna, Francisco Javier; Barceló Larran, Diego; Blázquez Agudo, Eva María; Checa Martín, José Luis; Cirujano González, Antonio; Pendás Pevida E et al. VIII Informe Adecco sobre Absentismo [Internet]. 2019. Disponible en: https:// www.adeccoinstitute.es/wp-content/uploads/2019/06/VIII-Informe-Absentismo. pdf

10. Páez AL, Abellán BR. Revisión bibliográfica. Implicaciones del presentismo en la productividad laboral del área de la salud. Medicina legal de Costa Rica [Internet]. 2015;32(1). Disponible en: https://www.scielo.sa.cr/scielo.php?script=sci_arttext\&pid=S1409-00152015000100018

11. World Health Organisation. Recommendations on digital interventions for health system strengthening [Internet]. 2019 [consultado 28 Mar 2021]. Disponible en: https://www.who.int/reproductivehealth/publications/digital-interventions-health-system-strengthening/en/

12. National Health Service. NHS Apps Library [Internet]. [consultado 28 Mar 2021]. Disponible en: https://www.nhs.uk/apps-library/

13. EU-OSHA. Category:E-tools - OSHWiki [Internet]. [consultado 28 Mar 2021]. Disponible en: https://oshwiki.eu/wiki/Category:E-tools

14. Bucci S, Schwannauer M, Berry N. The digital revolution and its impact on mental health care. In: Psychology and Psychotherapy: Theory, Research and Practice [Internet]. Wiley-Blackwell; 2019 [consultado 28 Mar 2021]. p. 277-97. Disponible en: https://bpspsychub.onlinelibrary.wiley.com/doi/10.1111/papt.12222

15. Howarth, Ana; Quesada, Jose; Silva, Jessica; Judycki, Stephanie; Mills PR. The impact of digital health interventions on health-related outcomes in the workplace: A systematic review. Digital Health [Internet]. Ene 2018 [consultado 28 Mar 2021];4:205520761877086. Disponible en: https://www.ncbi.nlm.nih.gov/pmc/articles/PMC6016571/ 
16. Rathbone, Amy Leigh; Prescott J. The use of mobile apps and SMS messaging as physical and mental health interventions: Systematic review [Internet]. Vol. 19, Journal of Medical Internet Research. JMIR Publications. 2017 [consultado 28 Mar 2021]. Disponible en: https://www.ncbi.nlm.nih.gov/pmc/articles/PMC5590007/

17. Joiner KL, Nam S, Whittemore R. Lifestyle interventions based on the diabetes prevention program delivered via eHealth: A systematic review and meta-analysis [Internet]. Vol. 100, Preventive Medicine. Academic Press Inc.; 2017 [consultado 28 Mar 2021]. p. 194-207. Disponible en: https://www.ncbi.nlm.nih.gov/pmc/articles/PMC5699208/

18. Spencer L, Rollo M, Hauck Y, MacDonald-Wicks L, Wood L, Hutchesson M, et al. The effect of weight management interventions that include a diet component on weight-related outcomes in pregnant and postpartum women: a systematic review protocol. JBI [Internet]. 1 Ene 2015 [consultado 28 Mar 2021];13(1):88-98. Disponible en: https://journals.Iww.com/jbisrir/Fulltext/2015/13010/The_effect_ of_weight_management_interventions_that.9.aspx

19. National Library of Medicine. Medical Subject Headings - Home Page [Internet]. [consultado 26 Ago 2021]. Disponible en: https://www.nlm.nih.gov/mesh/ meshhome.html

20. Mendeley Ltd. [Internet]. [consultado 14 Ago 2021]. Disponible en: https:// www.mendeley.com/search/

21. Suman A, Schaafsma FG, van Dongen JM, Elders PJM, Buchbinder R, van Tulder MW, et al. Effectiveness and cost-utility of a multifaceted eHealth strategy to improve back pain beliefs of patients with non-specific low back pain: A cluster randomised trial. BMJ Open [Internet]. 2019;9(12):1-9. Disponible en: https://bmjopen.bmj.com/content/9/12/e030879

22. van der Meij E, Anema JR, Leclercq WKG, Bongers MY, Consten ECJ, Schraffordt Koops SE, et al. Personalised perioperative care by e-health after intermediate-grade abdominal surgery: a multicentre, single-blind, randomised, placebo-controlled trial. The Lancet [Internet]. 2018;392(10141):51-9. Disponible en: https:// www.thelancet.com/journals/lancet/article/PIIS0140-6736(18)31113-9/fulltext

23. Blanquero J, Cortés-Vega MD, Rodríguez-Sánchez-Laulhé P, Corrales-Serra BP, Gómez-Patricio E, Díaz-Matas N, et al. Feedback-guided exercises performed on a tablet touchscreen improve return to work, function, strength and healthcare usage more than an exercise program prescribed on paper for people with wrist, hand or finger injuries: a randomised trial. Journal of Physiotherapy [Internet]. 2020;66(4):236-42. Disponible en: https://www.sciencedirect.com/science/article/pii/S1836955320301077?via\%3Dihub

24. Deady M, Glozier N, Calvo R, Johnston D, MacKinnon A, Milne D, et al. Preventing depression using a smartphone app: A randomized controlled trial. Psychological Medicine [Internet]. 2020; Disponible en: https://www.cambridge.org/core/journals/psychological-medicine/article/abs/preventing-depression-using-a-smar- 
tphone-app-a-randomized-controlled-trial/09131AFD5C2C36534ABDFEOF885FF7C4

25. Lokman S, Volker D, Zijlstra-Vlasveld MC, Brouwers EP, Boon B, Beekman AT, et al. Return-to-work intervention versus usual care for sick-listed employees: Health-economic investment appraisal alongside a cluster randomised trial. BMJ Open [Internet]. 2017;7(10). Disponible en: https://bmjopen.bmj.com/content/7/10/ e016348

26. van der Hout A, Jansen F, van Uden-Kraan CF, Coupé VM, Holtmaat K, Nieuwenhuijzen GA, et al. Cost-utility of an eHealth application 'Oncokompas' that supports cancer survivors in self-management: results of a randomised controlled trial. Journal of Cancer Survivorship [Internet]. 2021;15(1):77-86. Disponible en: https://link.springer.com/article/10.1007/s11764-020-00912-9

27. Notenbomer A, Roelen C, Groothoff J, van Rhenen W, Bültmann U. Effect of an eHealth intervention to reduce sickness absence frequency among employees with frequent sickness absence: Randomized controlled trial. Journal of Medical Internet Research [Internet]. 2018;20(10). Disponible en: https://www.cochranelibrary.com/central/doi/10.1002/central/CN-01650210/full

28. Volker D, Zijlstra-Vlasveld MC, Anema JR, Beekman AT, Brouwers EPM, Emons $\mathrm{WH}$, et al. Effectiveness of a blended web-based intervention on return to work for sick-listed employees with common mental disorders: Results of a cluster randomized controlled trial. Journal of Medical Internet Research [Internet]. 2015;17(5). Disponible en: https://www.ncbi.nlm.nih.gov/pmc/articles/PMC4468600/

29. Hutting N, Bart Staal J, Engels JA, Heerkens YF, Detaille SI, Nijhuis-Van Der Sanden MWG. Effect evaluation of a self-management programme for employees with complaints of the arm, neck or shoulder: a randomised controlled trial. Occupational and Environmental Medicine [Internet]. 2015;72(12):852-61. Disponible en: https://oem.bmj.com/content/72/12/852

30. Ebert DD, Heber E, Berking M, Riper H, Cuijpers P, Funk B, et al. Self-guided internet-based and mobile-based stress management for employees: Results of a randomised controlled trial. Occupational and Environmental Medicine [Internet]. 2016;73(5):315-23. Disponible en: https://oem.bmj.com/content/73/5/315.long

31. Bouwsma EVA, Huirne JAF, van de Ven PM, Vonk Noordegraaf A, Schaafsma FG, Schraffordt Koops SE, et al. Effectiveness of an internet-based perioperative care programme to enhance postoperative recovery in gynaecological patients: Cluster controlled trial with randomised stepped-wedge implementation. BMJ Open [Internet]. 2018;8(1):1-10. Disponible en: https://bmjopen.bmj.com/content/8/1/e017781

32. Beiwinkel T, Eißing T, Telle NT, Siegmund-Schultze E, Rössler W. Effectiveness of a web-based intervention in reducing depression and sickness absence: Randomized controlled trial. Journal of Medical Internet Research [Internet]. 2017;19(6):1-14. Disponible en: https://www.jmir.org/2017/6/e213/ 
33. Ghoshchi SG, de Angelis S, Morone G, Panigazzi M, Persechino B, Tramontano $\mathrm{M}$, et al. Return to work and quality of life after stroke in Italy: A study on the efficacy of technologically assisted neurorehabilitation. International Journal of Environmental Research and Public Health [Internet]. 2020;17(14):1-12. Disponible en: https://www.ncbi.nlm.nih.gov/pmc/articles/PMC7399919/

34. Wolf A, Fors A, Ulin K, Thorn J, Swedberg K, Ekman I. An eHealth diary and symptom-tracking tool combined with person-centered care for improving self-efficacy after a diagnosis of acute coronary syndrome: A substudy of a randomized controlled trial. Journal of Medical Internet Research [Internet]. 2016;18(2). Disponible en: https://www.jmir.org/2016/2/e40/

35. de Jong MJ, Boonen A, van der Meulen-de Jong AE, Romberg-Camps MJ, van Bodegraven AA, Mahmmod N, et al. Cost-effectiveness of Telemedicine-directed Specialized vs Standard Care for Patients With Inflammatory Bowel Diseases in a Randomized Trial. Clinical Gastroenterology and Hepatology [Internet]. 2020;18(8):1744-52. Disponible en: https://www.cghjournal.org/article/S15423565(20)30535-8/fulltext

36. Schaetz L, Rimner T, Pathak P, Fang J, Chandrasekhar D, Mueller J, et al. Employee and Employer Benefits From a Migraine Management Program: Disease Outcomes and Cost Analysis. Headache [Internet]. 2020;60(9):1947-60. Disponible en: https://headachejournal.onlinelibrary.wiley.com/doi/full/10.1111/head.13933

37. Mochari-Greenberger $H$, Andreopoulos E, Peters A, Pande RL. Clinical and Workplace Outcomes From a Virtually Delivered Cognitive Behavioral Therapy Program for Pain. Pain Practice [Internet]. 2020;20(4):387-95. Disponible en: https:// onlinelibrary.wiley.com/doi/10.1111/papr.12867

38. Anderson L, Kinsman S, Oberlander M. Postoperative Compliance and Return to Work After Rotator Cuff Repair: Value of an Interactive Online Rehabilitation Program Among Patients Treated Under Workers' Compensation. Orthopedics [Internet]. 2021;44(2):E197-202. Disponible en: https://journals.healio.com/ doi/10.3928/01477447-20201119-07

39. Engdahl $P$, Svedberg $P$, Lexén $A$, Bejerholm U. Role of a digital return-to-work solution for individuals with common mental disorders: Qualitative study of the perspectives of three stakeholder groups. JMIR Formative Research [Internet]. 2020;4(9):1-13. Disponible en: https://www.ncbi.nlm.nih.gov/pmc/articles/ PMC7527904/

40. Beaton MD, Hadly G, Babul S. Stakeholder Recommendations to Increase the Accessibility of Online Health Information for Adults Experiencing Concussion Symptoms. Frontiers in Public Health [Internet]. 2021;8(January):1-11. Disponible en: https://www.ncbi.nlm.nih.gov/pmc/articles/PMC7829503/

41. Gross DP, Asante A, Pawluk J, Niemeläinen R. A Descriptive Study of the Implementation of Remote Occupational Rehabilitation Services Due to the COVID-19 Pandemic Within a Workers' Compensation Context. Journal of Occupational Re- 
habilitation [Internet]. 2021;31(2):444-53. Disponible en: https://link.springer.com/ article/10.1007\%2Fs10926-020-09934-7

42. Collins DAJ, Harvey SB, Lavender I, Glozier N, Christensen H, Deady M. A pilot evaluation of a smartphone application for workplace depression. International Journal of Environmental Research and Public Health [Internet]. 2020;17(18):114. Disponible en: https://www.ncbi.nlm.nih.gov/pmc/articles/PMC7576478/

43. Karpman J, Gross DP, Manns P, Tomkins-Lane C. Do wearable fitness devices correlate with performance-based tests of work-related functional capacity? Work [Internet]. 2020;66(1):201-11. Disponible en: https://content.iospress.com/ articles/work/wor203164

44. Noben C, Evers S, Genabeek J van, Nijhuis F, de Rijk A. Improving a web-based employability intervention for work-disabled employees: results of a pilot economic evaluation. Disability and Rehabilitation: Assistive Technology [Internet]. 2017;12(3):280-9. Disponible en: https://www.tandfonline.com/doi/full/10.3109/ 17483107.2015.1135999

45. Streibelt M, Blume C, Thren K, Reneman MF, Mueller-Fahrnow W. Value of Functional Capacity Evaluation Information in a Clinical Setting for Predicting Return to Work. ACRM [Internet]. 2009;90(3):429-34. Disponible en: http://dx.doi.org/10.1016/j.apmr.2008.08.218

46. Fairbrother P, Ure J, Hanley J, McCloughan L, Denvir M, Sheikh A, et al. Telemonitoring for chronic heart failure: the views of patients and healthcare professionals - a qualitative study. JCN [Internet]. 1 Ene 2014 [consultado 14 Ago 2021];23(1-2):132-44. Disponible en: https://onlinelibrary.wiley.com/doi/ full/10.1111/jocn.12137

47. Nieuwenhuijsen K, Noordik E, van Dijk FJ, van der Klink JJ. Return to work perceptions and actual return to work in workers with common mental disorders. Journal of occupational rehabilitation [Internet]. Jun 2013 [consultado 14 Ago 2021];23(2):290-9. Disponible en: https://pubmed.ncbi.nlm.nih.gov/23124685/

48. Volker D, Zijlstra-Vlasveld. MC., Brouwers EP, van Lomwel AG, van der Feltz-Cornelis CM. Return-to-Work Self-Efficacy and Actual Return to Work Among LongTerm Sick-Listed Employees. Journal of occupational rehabilitation [Internet]. 22 Jun 2015 [consultado 14 Ago 2021];25(2):423-31. Disponible en: https://pubmed. ncbi.nlm.nih.gov/25354750/

49. Bolier L, Haverman M, Kramer J, Westerhof GJ, Riper H, Walburg JA, et al. An Internet-Based Intervention to Promote Mental Fitness for Mildly Depressed Adults: Randomized Controlled Trial. JMIR Publications [Internet]. 2013 [consultado 14 Ago 2021];15(9). Disponible en: https://www.ncbi.nlm.nih.gov/pmc/articles/ PMC3929047/ 